\title{
Complete Spacial Evaluation of the Preoperative Bariatric Patient New Insights into Body Composition
}

\author{
Stephen D. WOHLGEMUTH ${ }^{a, b}$, David STEFAN ${ }^{c}$ \\ ${ }^{a}$ Eastern Virginia Medical School, Norfolk VA, USA; \\ ${ }^{\mathrm{b}}$ Sentara Comprehensive Weight Loss Solutions, Norfolk VA, USA; \\ ${ }^{\mathrm{C}}$ Novaptus Systems Inc. Chesapeake, VA, USA \\ http://dx.doi.org/10.15221/13.340
}

Current techniques to evaluate the physical characteristics of the preoperative bariatric subject are generally limited to height, weight and perhaps anthropometric measurements such as waist and hips. By keeping the height and weight values, and using a commercial 3D whole body scanner and associated measurement software, one can provide a complete spacial evaluation of the subject, including excess volume, excess fat and fat density.

\section{Background}

Identifying the morbidly obese, at first glance, appears straightforward. From an observer's perspective, one perceives the morbidly obese as being extremely overweight, abnormal in both shape and size.

Categorizing and assessing the morbidly obese, however, has always been a challenge. Morbid obesity is defined as: 1). Being 100 pounds or more above your ideal body weight. 2). Or, having a Body Mass Index (BMI) of 40 or greater. 3). Or, having an excess body weight twice that of the norm for the height of the subject.

Subjects meeting one or more of these three conditions qualify as candidates for surgical weight loss.

There are certain subjects having a BMI of greater than 35 with one or more co-morbid condition (such as hypertension, Type 2 diabetes, high blood pressure, etc.) who can also qualify as a candidate for a surgical weight loss procedure. These subjects are not considered morbidly obese, but present additional assessment challenges for the bariatric surgeon.

Surgical weight loss procedures considered for all subjects who qualify would be gastric bypass or gastric sleeve device.

BMI has been a simple method of measuring obesity in the adult population. To compute the BMI value, one only needs an accurate scale and a stadiometer. Both instruments are inexpensive, readily available and easy to use. The formula for BMI is Weight, in $\mathrm{kg}$, divided by height, in meters, squared. Or, if using English units, one must multiply their weight, in pounds, by 703 , then divide that sum by their height, in inches, squared.

The classifications of BMI are indicated in Table $1^{1}$.

\begin{tabular}{|c|c|}
\hline & BMI \\
\hline Underweight & $<18.5 \mathrm{~kg} / \mathrm{m}^{2}$ \\
\hline Normal Weight & $18.5-24.9 \mathrm{~kg} / \mathrm{m}^{2}$ \\
\hline Overweight & $25-29.9 \mathrm{~kg} / \mathrm{m}^{2}$ \\
\hline Obese & $30-34.9 \mathrm{~kg} / \mathrm{m}^{2}$ \\
\hline Obesity with at least 1 Comorbid Condition & $35-35.9 \mathrm{~kg} / \mathrm{m}^{2}$ \\
\hline Morbid Obesity &.$>40 \mathrm{~kg} / \mathrm{m}^{2}$ \\
\hline
\end{tabular}

There are a number of issues with simply relying on the BMI value to determine obesity. Since the formula emphasizes height, squared, in the denominator, those subjects that are shorter are, in effect, penalized. The formula also doesn't take into account the physical condition of the subject. Bodybuilders, and those with a muscular build such as other sports athletes can have BMI values of 40 or more. Hence, simply observing the BMI value outside the context of viewing the subject could be highly deceiving. Finally, BMI gives no indication as to the physical shape of the subject. Morbidly obese subjects can generally be classified as gynecoid, or pear-shaped, android, or apple-shaped, or somewhere in between. Understanding where on the body excess fat is distributed helps assess the difficulty of the surgery, as well as the general health of the subject. 
Waist circumference evaluation assess the risks associated with obesity and is supported by research. Excess abdominal fat is an important, independent risk factor for disease. The measurement of waist-to-hip ratio provides no advantage over waist circumference alone, but can be used as a weak proxy for weight distribution. Table 2 outlines the relationship between BMI and waist circumference for defining risk. ${ }^{2}$

\begin{tabular}{|c|c|c|c|c|}
\hline & \multirow[t]{2}{*}{$\begin{array}{c}\text { BMI } \\
(\mathrm{kg} / \mathrm{me})\end{array}$} & \multirow[t]{2}{*}{ Obesity Class } & \multicolumn{2}{|c|}{$\begin{array}{l}\text { Disease Risk* } \\
\text { (Relative to Normal Weight } \\
\text { and Waist Circumference) }\end{array}$} \\
\hline & & & $\begin{array}{c}\text { Men } \leq 40 \text { in }(\leq 102 \mathrm{~cm}) \\
\text { Women } \leq 35 \text { in }(\leq 88 \mathrm{~cm})\end{array}$ & $\begin{array}{l}>40 \text { in }(>102 \mathrm{~cm}) \\
>35 \text { in }(>88 \mathrm{~cm})\end{array}$ \\
\hline Underweight & $<18.5$ & & - & - \\
\hline Normalt & $18.5-24.9$ & & - & - \\
\hline Overweight & $25.0-29.9$ & & Increased & High \\
\hline Obesity & $\begin{array}{l}30.0-34.9 \\
35.0-39.9\end{array}$ & $\begin{array}{l}\text { I } \\
\text { II }\end{array}$ & $\begin{array}{l}\text { High } \\
\text { Very High }\end{array}$ & $\begin{array}{l}\text { Very High } \\
\text { Very High }\end{array}$ \\
\hline Extreme Obesity & $\geq 40$ & III & Extremely High & Extremely High \\
\hline
\end{tabular}

While there are standard methodologies for measuring waist circumference of morbidly obese individuals, an internal study performed as part of a quality improvement program for our clinical practice demonstrated that several trained nurses who independently measured morbidly obese subjects' waist circumferences had measurement results with wide margins of error.

There is a limit to what can be done in assessing the morbidly obese subject using the common tools of the scale, stadiometer and tape measure.

More advanced methods of assessing total and regional adiposity have been employed. Two main groups are often cited: Those that are used mainly in research laboratories as reference methods, including whole-body $\mathrm{K}$ counting, neutron activation analysis, hydrometry, underwater weighing/air plethysmography, dual-energy x-ray absoptiometry, multicomponent model, and imaging methods such as computerized axial tomography and magnetic resonance imaging; and those that are applicable to field settings such as bioimpedance analysis. ${ }^{3}$

The definition of anthropometry, according to Merriam-Webster, is the study of human body measurements especially on a comparative basis. Morbidly obese bariatric subjects have been traditionally difficult to measure manually, and for the most part, other than measuring waist and hips, often go through the surgical weight loss evaluation process without being thoroughly measured.

\section{Methods}

We began using a 3D whole body scanner to measure preoperative bariatric patients in late 2005 as a convenient method to remedy the lack of accurate and thorough anthropometric documentation. The 3D body scanner, a commercially available unit, was adapted from the custom-tailored clothing industry, where it had been successfully utilized for a number of years. A typical example would be for a subject to stand in a fixed position in the scan chamber. An accurate 3D body model would then be produced, and a predefined measurement extraction profile would be used to extract measurements from the body model to create a customized garment (pants, skirt, shirt, etc.).

At that time, the scanning unit utilized white light for projection and multiple cameras at fixed angles within a darkened scan chamber to capture images of the subject standing inside. Once captured, these images were then assembled into an accurate 3D body model. Scanner camera technology improvements have replaced white light projection cameras with near-field infrared sensors, but the process of capturing images from cameras at various fixed angles to produce a measurable 3D body model remains the same.

Flexible measurement programmability allows multiple measurements to be extracted from the same 3D body model. 
The first set of measurements simply concentrated on common measurements of which subjects would have familiarity. These included circumferential measurements such as the neck, chest, bust, waist, abdomen, hips, thighs and calves. Figure 1 depicts a 3D body model of a preoperative bariatric subject and the common measurements extracted.

\section{Circumferential Measurements}
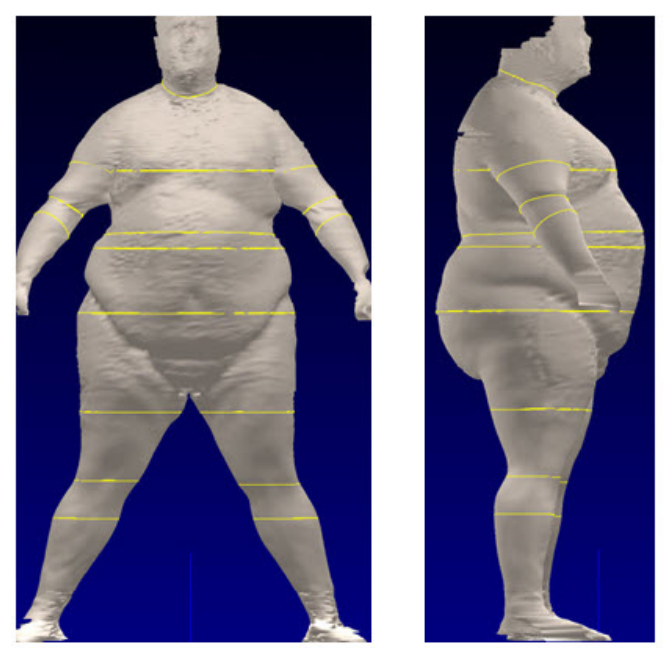

\begin{tabular}{l|r} 
Measurements, Inches & Pre-op \\
\hline Neck_Full & 18.84 \\
\hline Bust_Full & 57.15 \\
\hline Waist_Full & 58.11 \\
\hline Abdomen_Full & 61.11 \\
\hline Hips_Full & 67.05 \\
\hline Right_Biceps & 19.08 \\
\hline Right_Elbow & 14.12 \\
\hline Right_Forearm & 13.75 \\
\hline Left_Biceps & 17.61 \\
\hline Left_Elbow & 12.82 \\
\hline Left_Forearm & 13.14 \\
\hline Thigh_Left & 30.30 \\
\hline Thigh_Right & 31.37 \\
\hline Knee_Left & 18.97 \\
\hline Knee_Right & 19.26 \\
\hline Calf_Left & 20.03 \\
\hline Calf_Right & 20.27 \\
\hline
\end{tabular}

Figure 1. 3D Body Model of Preoperative Bariatric Subject with Common Measurements

It should be noted that each associated measurement has a height component, as measured from the floor of the scanner.

The 3D scanner offered a convenient and repeatable method to accurately document the subject, not only preoperatively, but also at various intervals after the bariatric surgery. For the first time, subjects could receive a "snapshot" of their physical measurements, as well as a profile and side view of their physical shape. This has opened up avenues to be explored, though not in this paper, of enhanced Body Image Assessment techniques.

The scanner software is capable of producing linear, circumferential, surface area and volume measurement information through the programming of measurement extraction profiles. Information can be displayed in $\mathrm{mm}, \mathrm{cm}$ or inches.

BMI, as noted earlier, plays a large role in assessing whether an adult is considered morbidly obese. In general, the higher the BMI value, the more obese the subject. In practical surgical terms, this has limitations. A male subject who presents for bariatric surgery that has a relatively low BMI but a large degree of central obesity can be a much more challenging operation than a significantly larger female with greater BMI who has the majority of her excess fat distributed about her hips.

Since the scanner measurement software was capable of calculating surface area and volume, the effort was made to explore whether there was a relationship between these components that could offer a better insight into the subject's obese condition. A measurement template was created to calculate the torso volume and torso surface area. The torso was defined by the scanner software to be from the back of the neck to the "crotch-point" or area where the torso separated from the legs. This definition of the torso included the hips and buttocks. Thus the torso could act as a proxy for the body of the overall subject and could be used to determine how much "space" the subject occupies independent of overall height and weight.

Figure 2 depicts how the scanner software defines the torso. Once the torso length has been defined, the software then slices the torso into $1 \mathrm{~cm}$ segments. Every segment has its own circumference. The surface area for each segment is calculated, and summed over all torso segments to calculate torso surface area.

Each segment is then closed, and the volume is calculated. Summing each segment's volume yields the torso volume. 
The results can be presented as a ratio, known as the Torso Volume/Torso Surface Area Ratio, or TVSA.

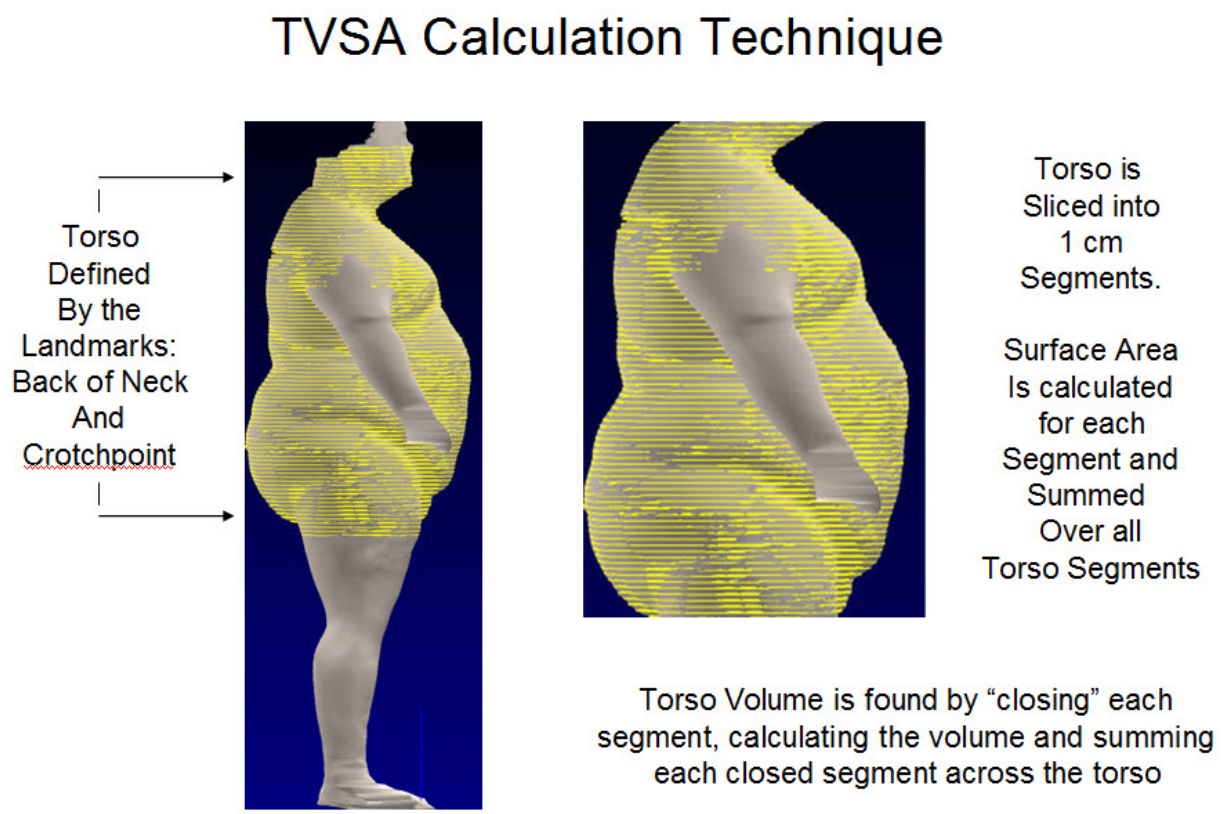

Figure 2. Calculating the Torso Surface Area and Torso Volume

There is a weak interrelation between BMI and TVSA in that TVSA contains a height component, torso height, which is a subset of overall height. Figure 3 depicts the example subject's BMI and TVSA ratio.

\title{
TVSA Calculation Results and BMI Comparison
}

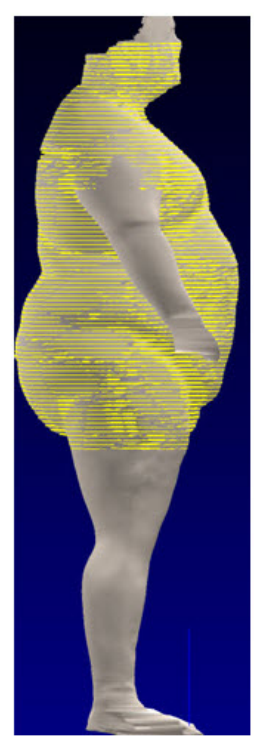

\author{
Torso Volume: 130033 cubic cm \\ Torso Surface Area: $11831 \mathrm{sq} \mathrm{cm}$ \\ Torso Vol/Torso SA $=10.99$
}

Subject Weight: 384 lbs

Subject Height: 70 in

Subject BMI: 55

Figure 2. BMI and TVSA Ratio for Example Subject

But the TVSA does not have a weight component. This begins to eliminate bias shown in the BMI with respect to the overall weight and height association of the subject. A practical example is shown in Figure 4. Here we have two individuals with the same height and weight and hence the same BMI. The subject on the left is an individual that is beginning to show signs of centralized obesity. The subject on the right is an amateur bodybuilder. 


\section{The Utility of the TVSA vs BMI}

The TVSA determines the "space" an individual occupies vs. the height and weight of the individual

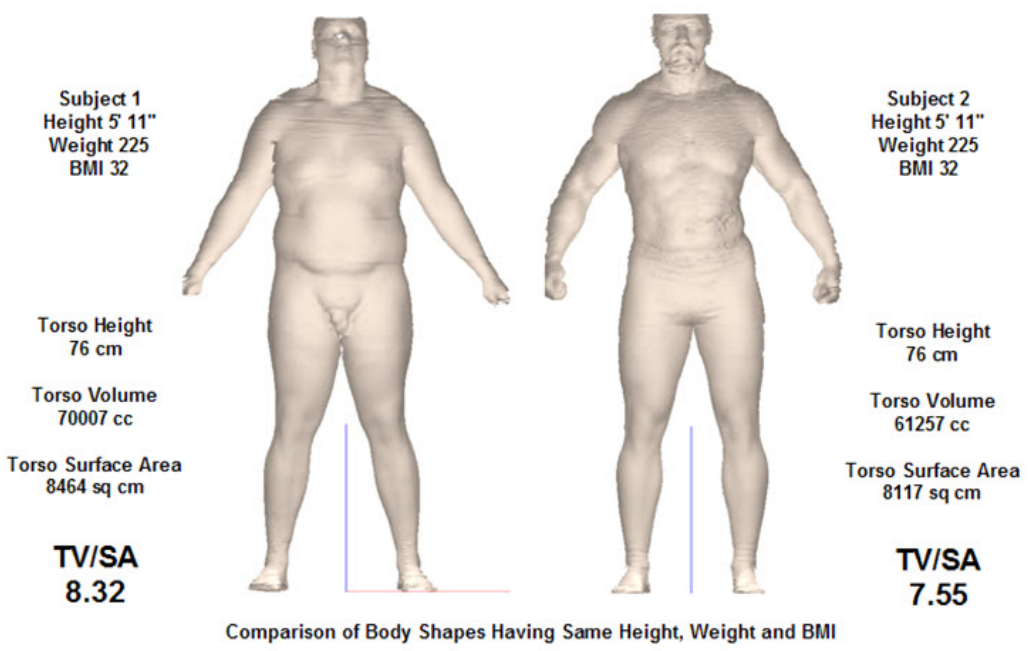

Figure 4. TVSA and BMI for Two Individuals Having the Same Height and Weight

There is a relationship between torso surface area and torso volume, which can be observed from the subjects in Figure 4. In general, the more obese the subject the greater their torso volume is as compared to their torso surface area. The less obese the subject, the greater the torso surface area is compared to their torso volume.

A way to conceptualize this is to imagine a single sheet of paper. It has relatively small volume (because of the thickness) compared to a rather large surface area (front and back of the sheet). As you begin to add more sheets of paper, the volume increases in disproportion to the surface area. Assuming perfectly square pieces of paper, you will eventually get to a perfect cube in which both the surface area and volume are in true proportion.

A practical use in the evaluation of bariatric subjects using the TVSA can be seen in Figure 5 and Figure 6 . Here can be observed morbidly obese subjects that have similar BMI values for both the high end of the BMI range and the lower end of what would be considered a morbidly obese BMI with at least 1 co-morbid condition.

\section{Practical Uses for the TVSA for Bariatric Evaluation}

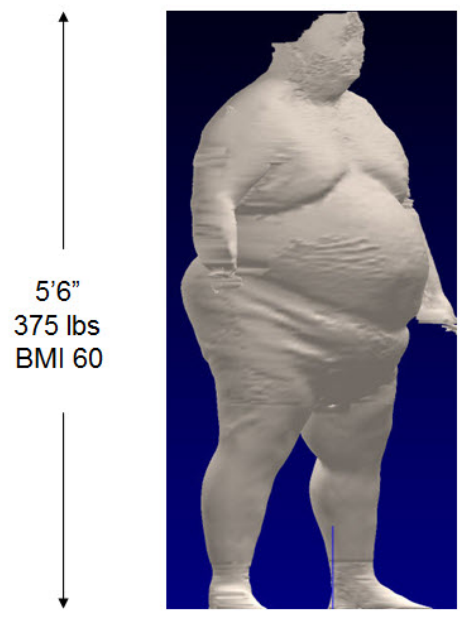

TVSA: 10.52

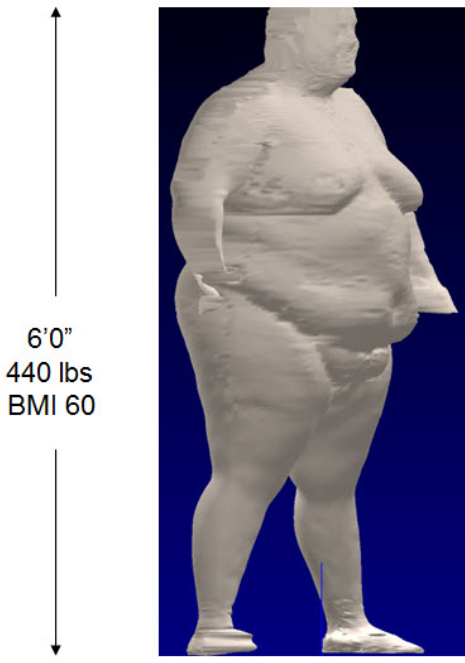

TVSA: 11.47

Figure 5. Dramatically Different Weight and Height, Same BMI, Different TVSA 


\section{Differentiating Between Lower BMI's}

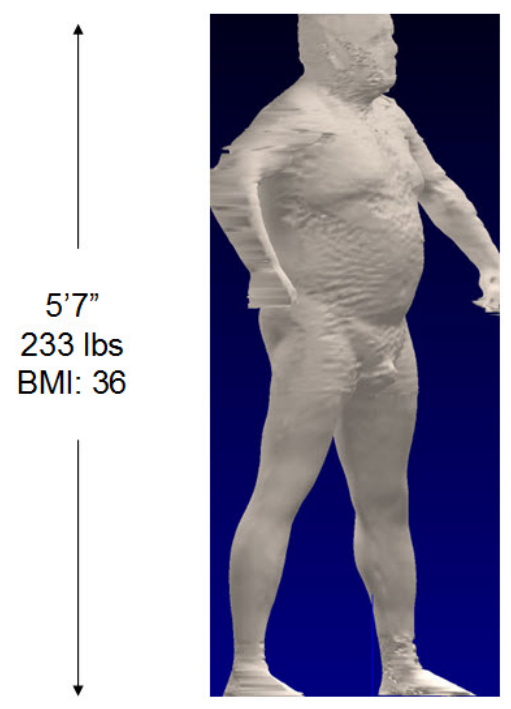

TVSA: 8.09

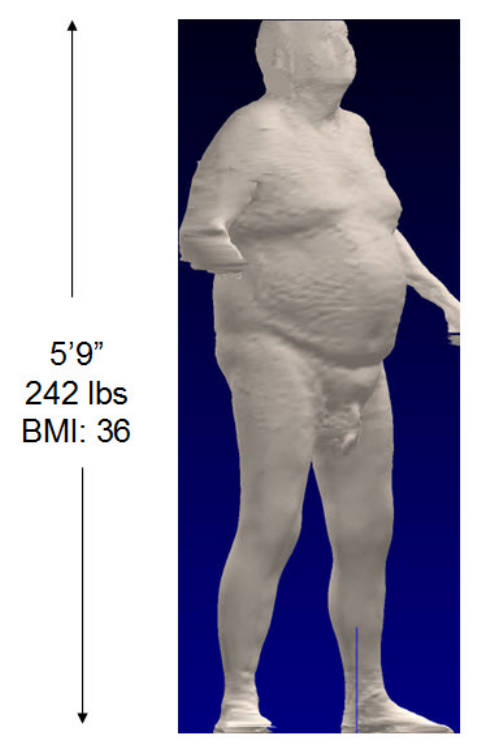

TVSA 8.79

Figure 6. Similar Weight and Height, Same BMI, Different TVSA

The TVSA ratio is a relative ratio. In general it can be stated that those who have a larger TVSA ratio are more obese than those that have a lesser TVSA ratio given relative overall sizes. To make the TVSA an absolute ratio, the specific torso height must be introduced. In this way, one can compare the TVSA of one subject directly to the TVSA of another subject that has the same torso height.

Figure 7 depicts torsos that have the same torso height, $64 \mathrm{~cm}$. The torsos range from ectomorph, to endomorph to morbidly obese.

\section{Comparison of Subjects with Same Torso Height}

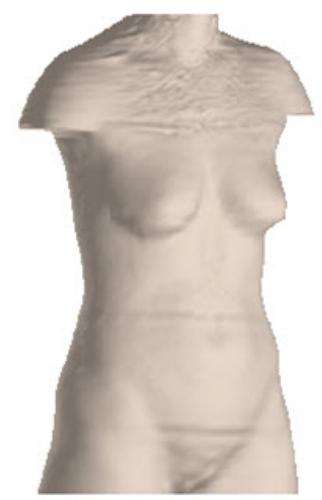

Ectomorph

Torso Volume $31860 \mathrm{cc}$ Torso Surface Area $5391 \mathrm{sq} \mathrm{cm}$ TVSA $=5.91$

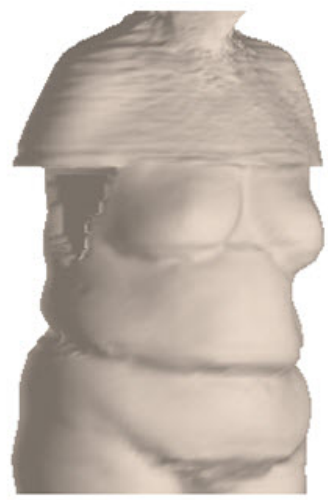

Endomorph

Torso Volume $55921 \mathrm{cc}$ Torso Surface Area $7135 \mathrm{sq} \mathrm{cm}$ TVSA $=7.84$

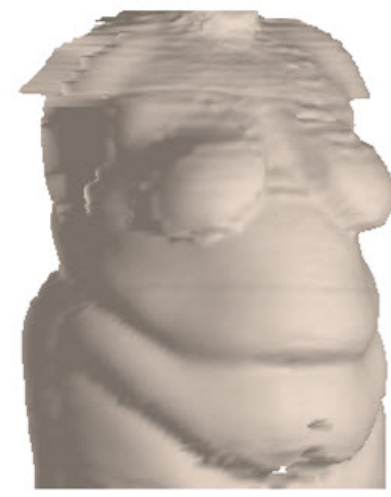

Morbidly Obese

Torso Volume $84858 \mathrm{cc}$ Torso Surface Area $8667 \mathrm{sq} \mathrm{cm}$ TVSA $=9.79$

Each Torso Height is $64 \mathrm{~cm}$

Figure 7. Fixed Torso Height TVSA Comparisons

Fixing the torso height in the example allows one clearly to see that volume and surface area do not change proportionally as a subject becomes obese, and for a fixed torso height the higher the TVSA value, the more obese the subject. 
The TVSA appears to be more advanced than BMI in that it takes into account two-dimensional and three-dimensional information that could not be previously conveniently been made available.

The TVSA still does not bear any shape information. However, the software measurement that provides torso volume and torso surface information can be used to mathematically classify the shape of the morbidly obese patient.

In general, morbidly obese patients can be placed into three categories: Android, whereby most of the excess fat is centered around the waist; gynecoid, where much of the excess fat is distributed around the hips, and mixed, where the shape of the subject does not offer any clear visual distinction between the two extremes.

Figure 8 shows the three general classifications for morbidly obese subjects.

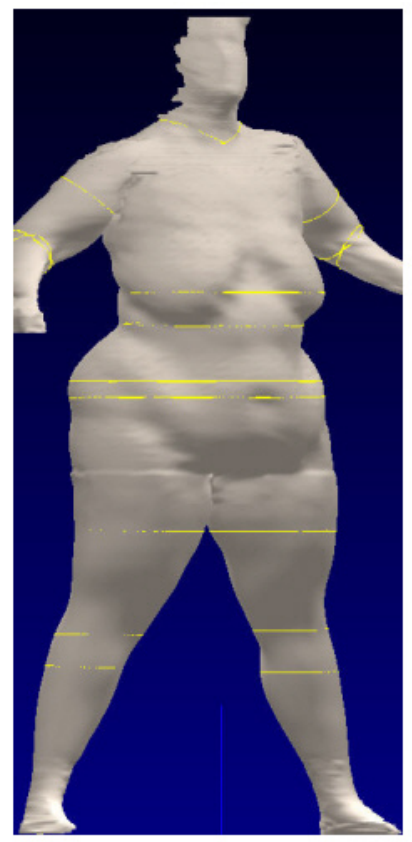

Gynecoid Shape

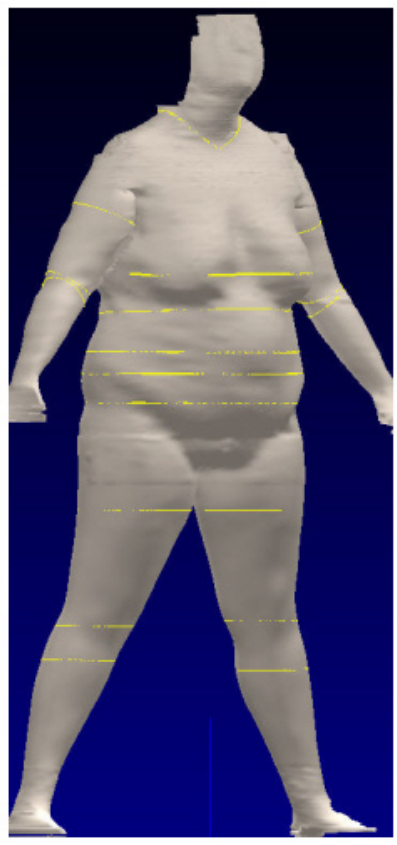

Mixed Shape

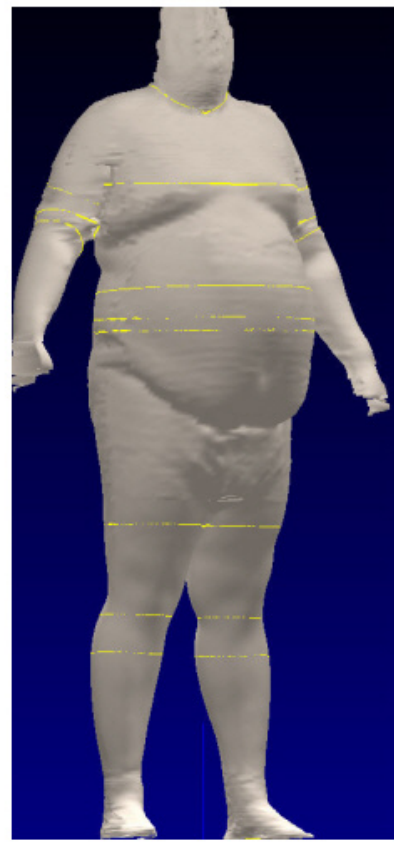

Android Shape

Figure 8. Common Shapes of Morbidly Obese Subjects

To mathematically determine the shape of the morbidly obese subject, the software that calculates the torso surface area and volume of each segment can be searched to find the torso segment that has the largest circumference and the height associated with that particular segment. Care must be taken that this circumference is at, near or below the waist height measurement. Subjects with broad chests can have a maximum torso slice circumference above the waist. This is relatively rare in morbidly obese subjects, but becomes commonplace when subjects approach a "normal" shape.

First, the overall torso height is determined. The maximum torso circumference segment represents the height on the torso where maximum fat distribution exists. Surface area and volume are calculated for the segments from the back of the neck down to the maximum torso segment, and divided by the overall TVSA. This yields the "Primary Shape" of the subject.

The result is a dimensionless number that represents how the torso has its space distributed. The larger this number, the greater the distribution is around the hips. The lower the number, the maximum distribution is closer to the waist.

Figure 9 helps to conceptualize these findings. If the maximum torso slice circumference is near the median of the torso, presumably the maximum fat distribution is around the waist. If the maximum torso slice circumference is closer to the bottom of the torso, the maximum fat distribution is around the hips. The resulting number is range bound between 0.50 (a perfect android where volume and surface area above the median is the same as volume and surface area below the median) and 1.0, where maximum volume and surface area occur at the bottom of the torso. 


\section{Shape Determination Concepts - Primary Shape}

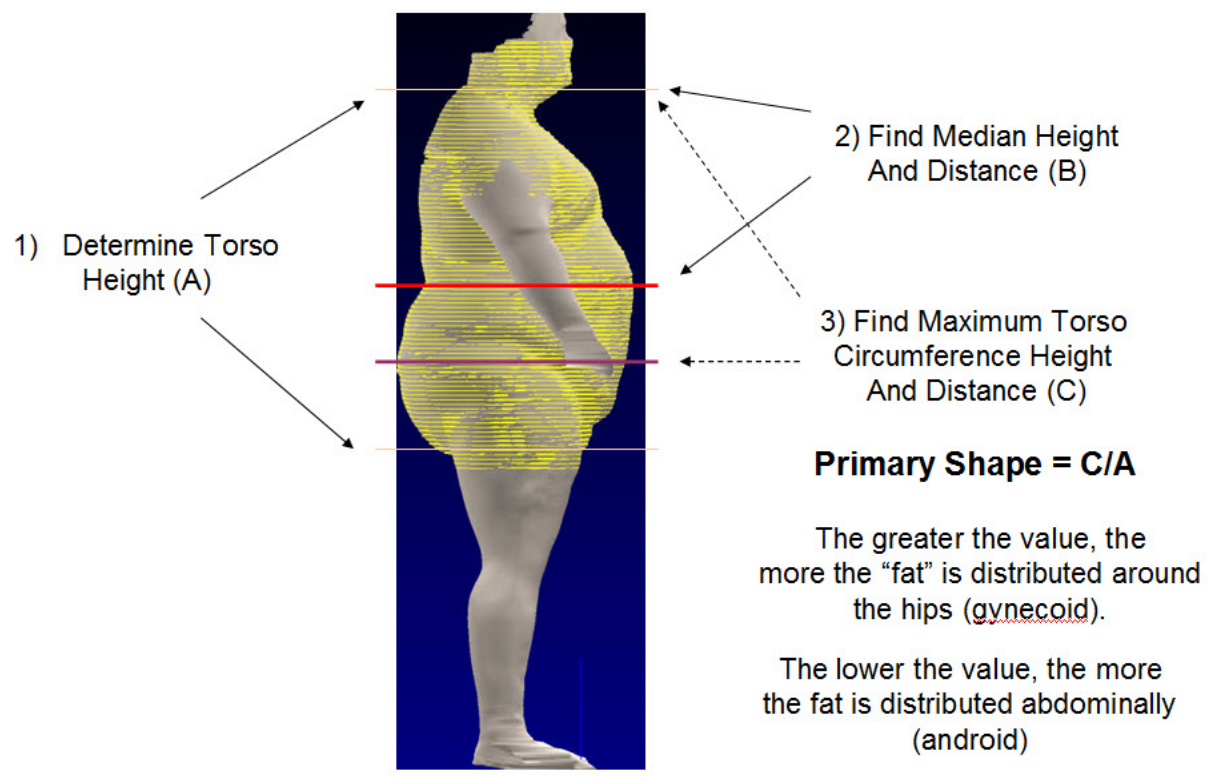

Figure 9. Primary Shape Determination Concept

Once the primary shape is determined, a secondary indicator, the "Shape Tendency", can be calculated. The shape tendency represents the surface area and volume from the back of the neck to the maximum torso circumference height, divided by the surface area and volume from the maximum torso circumference height to the crotch-point. This secondary indicator does not have meaning without the context of the primary shape indicator. The primary shape indicates what shape the subject is. The shape tendency indicates the degree, or trend within the specific primary shape categorization.

Figure 10 outlines the calculation for shape tendency. It also outlines the Shape Descriptor Table that gives specific numbers that mathematically classify the shape, and the degree of the shape of the morbidly obese subject.

\section{Shape Determination Values - Shape Tendency}

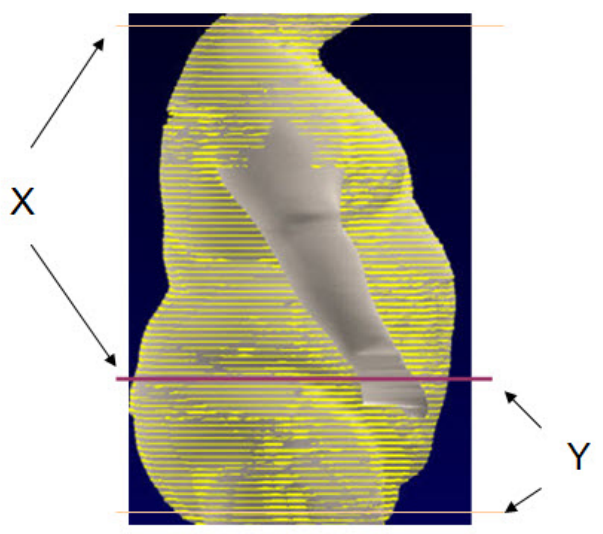

1) Determine distance between back of neck height maximum circumference height $(X)$

2) Determine distance between torso maximum circumference height and crotchpoint $(Y)$

\section{Shape Tendency:}

Divide the volume/surface area in Segment $Y$ Into volume/surface area in Segment $X$

The Shape Descriptor Table

\begin{tabular}{c|c|c} 
& Primary Shape & Shape Tendency \\
\hline Android & 0.69 or lower & higher value tends android \\
\hline Mixed & $\mathbf{0 . 7 0 \text { to } 0 . 7 9}$ & lower value tends gynecoid \\
\hline Gynecoid & $\mathbf{0 . 8 0}$ or higher & lower value tends gynecoid
\end{tabular}

Figure 10. Calculating the Shape Tendency and The Shape Descriptor Table 
Figure 11 depicts the results of applying these Shape Descriptor formulas to the subjects in Figure 8.

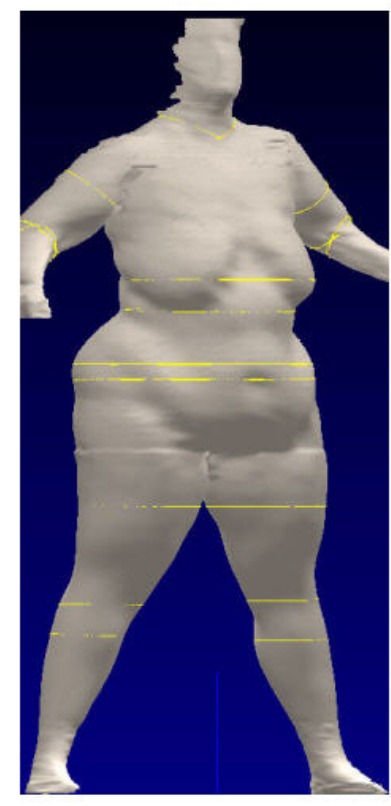

Gynecoid Shape $0.86,016$

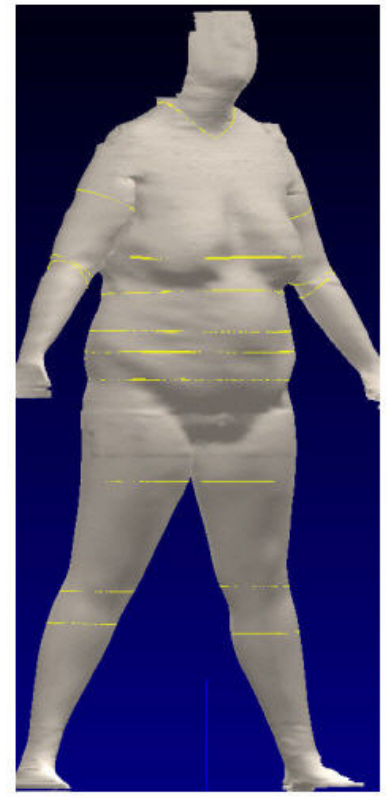

Mixed Shape $0.75,0.34$

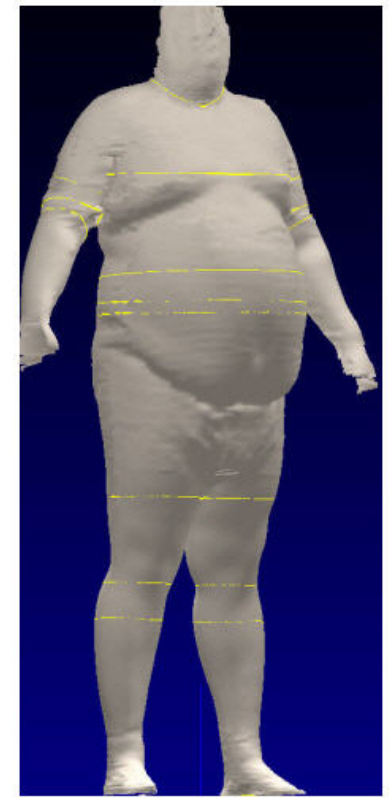

Android Shape $0.63,0.45$

Figure 11. Shape Descriptors Applied to Common Morbidly Obese Body Shapes

By utilizing the programmable software measurement capabilities of the scanner, we have started to define morbid obesity in terms of the overall size, or "space" the subject occupies, and have computed a method to mathematically determine the physical shape of the morbidly obese subject. These two elements, taken collectively and complemented with BMI, yield much more insight into the obese condition.

The third measurement capability offered by the scanner is the ability to calculate "bulk" volume of the subject. Heretofore, finding the volume of a subject required specialized equipment such as a BOD $P O D$, or dunking the subject into a water tank to measure the known water displacement.

The scanner software essentially compiles photographs from cameras at fixed angles within the scanner chamber. The photographs are "stitched" together, then reduced to form a body model. In the process of forming the body model, the scanner searches for known landmarks on the body, such as the back of neck, the suprasternal notch, small of back and separation between arms and legs from the torso.

The resultant body model consists of seven body "parts": Torso, including the head, left and right arms, left and right hands, and left and right legs.

The body model is comprised of thousands of points on the surface of the body parts, as represented by Cartesian coordinates, with $(X=0, Y=0, Z=0)$ being the center spot on the floor of the scanner. To calculate surface area of a body part, these points are connected into triangles. The surface area of each triangle is calculated, then summed to yield the surface area of the particular body part on the body model. To calculate volume, the triangles are converted to polygons. The volume of each polygon within the body part is calculated then summed to yield overall body "bulk" volume. Figure 12 displays "bulk" volume and surface area calculations as provided by the scanner measurement software. 


\section{Introducing Bulk Volume and Body Part Surface Area}
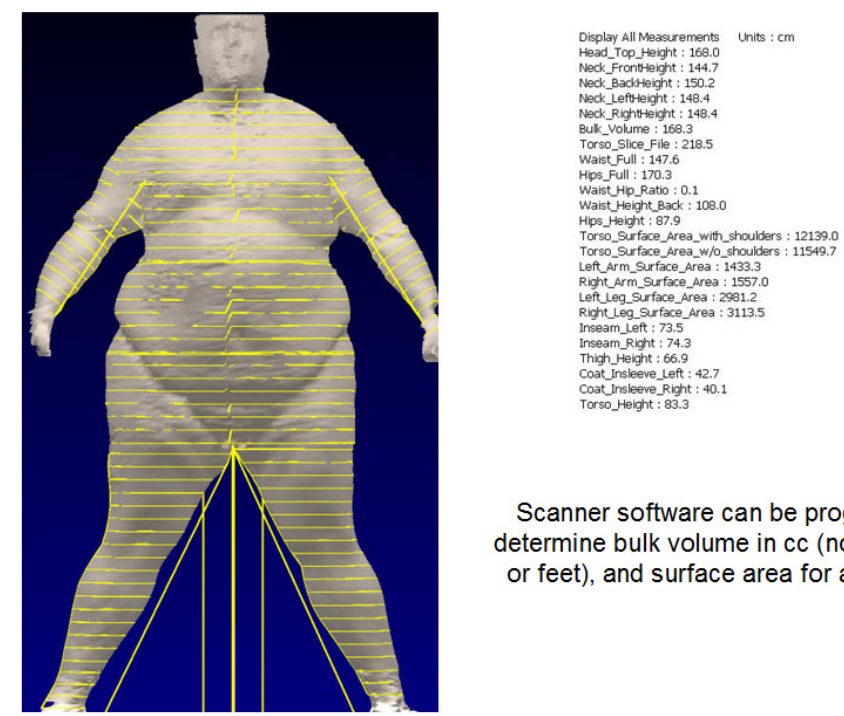

Scanner software can be programmed to determine bulk volume in cc (no head, hands or feet), and surface area for all body parts

Figure 12. Example Calculation of "Bulk" Volume and Body Part Surface Area

The current, well-accepted method of evaluating the progress and success of a bariatric surgical procedure is to determine the excess weight of the preoperative subject. A bariatric surgery is said to be successful (all other things considered) if the subject loses $75 \%$ of their excess weight over a period of 1 year.

Today, the patient is weighed prior to surgery. A table, typically an actuarial insurance table, is referenced that indicates "Ideal Weight" based on items such as height, gender, age and sometimes ethnicity.

Given the excess weight value from table look-up, and the bulk volume measurement from the scanner, a new parameter, "Excess Volume", in cc, can be calculated.

The calculations are straightforward. If one knows the bulk volume of the subject, as given from the scanner, and knows both the current weight (in $\mathrm{kg}$ ) and excess weight (in $\mathrm{kg}$, from table lookup) one can find the excess volume by dividing the bulk volume by the current weight. This termed has been coined "Bulk Mass". The bulk mass is then multiplied by the excess weight.

The kg units cancel and you are left with excess volume, in cc. Figure 13 outlines this process.

\section{Excess Volume Calculation Example}

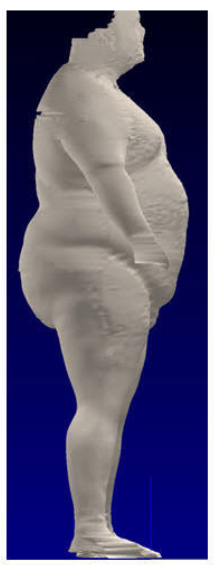

$178 \mathrm{~cm}, 174 \mathrm{~kg}$ BMl: 55
Bulk Volume: 168300 cc

Current Weight: $174 \mathrm{~kg}$

Ideal Weight: $78 \mathrm{~kg}$ (from table lookup)

Weight to Lose: $97 \mathrm{~kg}$

$\%$ Excess Weight: $55 \%$

"Bulk Mass" = Bulk Volume $/$ Current Weight $=966 \mathrm{cc} / \mathrm{kg}$

Volume to Lose: Bulk Mass $\mathrm{x}$ Weight to Lose $=93354 \mathrm{cc}$

\% Excess Volume: $55 \%$ (note \% is same as initial condition)

Figure 13. Calculating Excess Volume, in cc 


\section{Discussion}

Thus, for the first time, we now have a new item to explore, excess volume, in cc. Volume and weight can be combined to form a density, or its inverse, a mass. Density is normally defined in $\mathrm{kg} / \mathrm{cc}$, or how much a specific something weighs within a uniform space, in this case a cubic centimeter.

If we assume that excess volume, like its counterpart, excess weight, is related primarily to excess fat, then we can use the well-documented value of the density of human fat as considered to be 0.918 $\mathrm{gm} / \mathrm{ml}$. Since $1 \mathrm{ml}$ is, by definition, $1 \mathrm{cc}$, human fat density can be considered to by $0.918 \mathrm{gm} / \mathrm{cc}$. Converting this value to $\mathrm{kg} / \mathrm{cc}$ yields $0.000918 \mathrm{~kg} / \mathrm{cc}$ as a human fat density.

This excess volume parameter can be utilized in various ways. For instance, we have defined the Bariatric Mass as excess volume divided by the weight of a bariatric subject, the result is a number in $\mathrm{cc} / \mathrm{kg}$. We also could define a number of other volume related parameters now that this information has been made available for use.

Multiplying this Bariatric Mass, for instance, in $\mathrm{cc} / \mathrm{kg}$ by the human fat density in $\mathrm{kg} / \mathrm{cc}$ yields a dimensionless number that could be considered to be an adiposity index of a bariatric subject.

The volume related components are now being rigorously investigated. Since we have determined the spacial relationships of morbidly obese subjects via the TVSA, and have mathematically determined the shape of the patient using the Shape Descriptors, there are various "filters" that can be applied to these newly created adiposity indices that could determine the degree of centralized obesity of a morbidly obese subject.

Figure 14 summarizes the various scanner-produced inputs as well as traditional inputs such as weight and height. These various inputs can now be combined to offer a complete spacial definition of the morbidly obese subject.

\section{Input - Output}

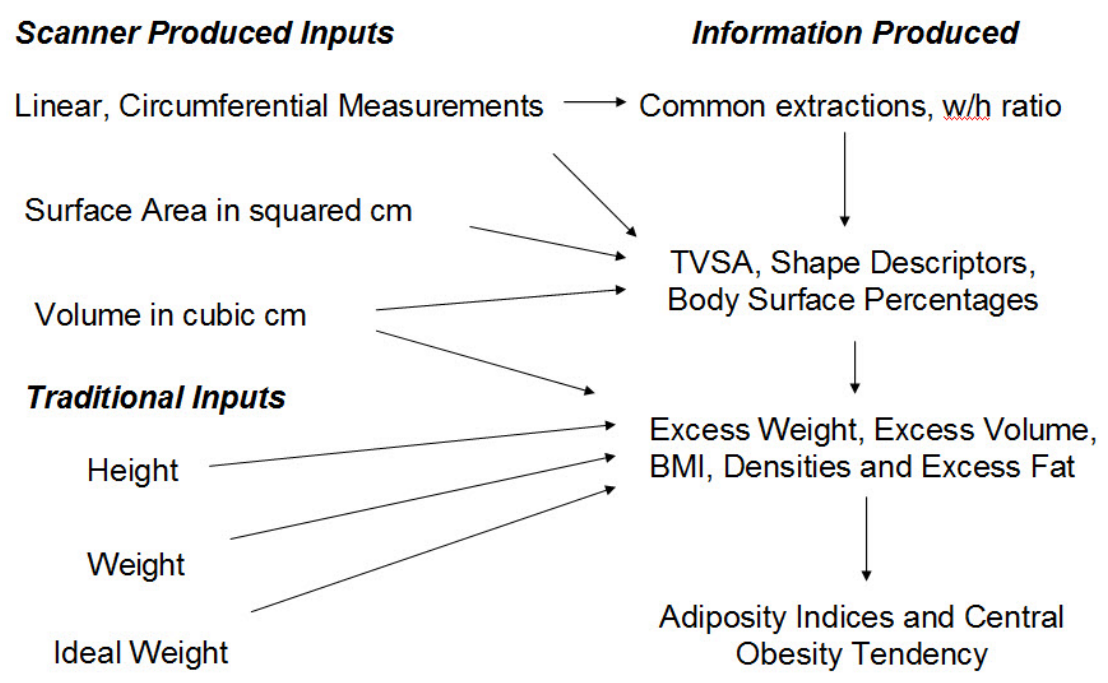

Figure 14. Complete Set of Multidimensional Inputs to Define the Morbidly Obese

\section{Conclusion}

The practical use of the scanner as a tool for documenting the preoperative and postoperative bariatric patient has proven to be effective in a clinical practice environment, simply by yielding accurate and repeatable common circumferential measurements on subjects who heretofore were difficult to comprehensively measure.

The additional information that can be provided, including volume and surface area of the torso and torso slice segment heights allowed the ability to create a ratio, the TVSA ratio that is weight independent and offers additional insight into the obese condition beyond BMI. 
The convenient calculations of "bulk volume", combined with traditional table lookup to find excess weight has yielded a new parameter to examine: "Excess Volume". Excess volume can be combined with various weight information to define new "mass indices".

Using the known density of human fat, in $\mathrm{kg} / \mathrm{cc}$, dimensionless adiposity indices can be investigated. These adiposity indices define generalized excess fat within the morbidly obese subject.

By applying appropriate filters to these newly created adiposity indices, such as the TVSA, primary shape, waist circumference or a combination of these filters, we postulate that "risk indication" numbers can be developed that relate to morbidly obese subjects that have a preponderance of comorbid conditions.

Identifying the set of markers associated with morbidly obese subjects that have comorbid conditions might then be applied to non-morbidly obese subjects who are at risk of developing comorbidities, should they continue on to the morbidly obese state.

By identifying these individuals who are at risk of developing these conditions prior to the morbidly obese state, preventative measures such as severe calorie restricted diets, exercise regimens or even preemptive surgical weight loss might be considered, ultimately leading to healthier lifestyles and less expensive long-term medical costs.

\section{References}

1. Adapter from 1998 National Institutes of Health Clinical Guidelines on the Indentification, Evaluation, and Treatment of Overweight and Obesity in Adults.

2. Adapted from Preventing and Managing the Global Epidemic of Obesity. Report of the World Heath Organization Consultation on Obesity. "WHO, Geneva, June 1997".

3. Bray, G, Bouchard, C. Evaluation of Total and Regional Adiposity. Handbook of Obesity pp 33. Marcel-Dekker, Inc. New York. 2004. 